\title{
The way to the heart is through the stomach; PPI's, aspirin, and gastric radiotracer activity
}

\author{
Stephen A. Bloom, MD, FASNC, ${ }^{\text {a }}$ and Randall C. Thompson, MD, FASNC ${ }^{b}$ \\ a Midwest Heart \&Vascular Specialists, Overland Park, KS \\ b St. Luke's Mid America Heart Institute, Kansas City, MO
}

Received Dec 4, 2019; accepted Dec 5, 2019

doi: $10.1007 / \mathrm{s} 12350-019-02010-5$

\section{See related article, pp. 1976-1985}

Increased gastric wall radiotracer activity (GWA) on SPECT myocardial perfusion imaging studies (MPI), as opposed to the more common hepatobiliary activity and reflux of activity into the stomach, is not well studied and appears to be uncommon, but can interfere with image interpretation. ${ }^{1-6}$ GWA can lead to false positive or false negative MPI results through overlap with the inferior left ventricle, amplified by the effect of Compton scatter and ramp filtering. The association of gastric wall activity and proton pump inhibitors has been described by several authors previously, although possible mechanisms have been untested, and selection bias has been considered possible. ${ }^{1-6}$ While a great many patients referred for SPECT MPI testing take proton pump inhibitors, this interfering activity seems to occur in the minority of them, and it appears to be a clinical problem in fewer still. Nevertheless, the images of occasional patients might be affected severely. ${ }^{6}$

In this article of the journal, Rose et al investigated a possible mechanism for this association of PPIs with gastric wall activity. ${ }^{7}$ They hypothesized that prolonged PPI use, acting through hypergastrinemia, leads to Cox2 expression and increased prostaglandin synthesis in the gastric mucosa and could promote increased radiotracer uptake via increased perfusion, increased mass of mitochondrion-rich parietal cells, or both. Concurrent use of aspirin would be predicted to inhibit those affects. This observational study of 319 patients undergoing

Reprint requests: Stephen A. Bloom, MD, FASNC, Midwest Heart \&Vascular Specialists, $5100 \mathrm{~W}$ 110th Street, 2nd floor, Overland Park, KS 66211; SBloomks@gmail.com

J Nucl Cardiol 2021;28:1986-7.

$1071-3581 / \$ 34.00$

Copyright (c) 2020 American Society of Nuclear Cardiology. technetium-99m sestamibi rest/stress MPI was divided into four subgroups: those taking neither a PPI nor aspirin, those taking aspirin and no PPI, those taking a PPI and no aspirin, and those taking both a PPI and aspirin. There was strong evidence that taking a PPI increases the odds of clinically significant gastric wall MIBI uptake. Taking the combination of PPI and aspirin also increased the odds of gastric uptake but to a lesser extent. ${ }^{7}$ Their data are therefore compatible with their hypothesized mechanism of PPIs increasing gastric activity.

\section{HOW FREQUENT IS THIS A CLINICAL PROBLEM WHAT CAN BE DONE ABOUT IT WHEN IT IS PRESENT?}

SPECT image interference from gastric wall activity seems to be uncommon. For example, three prior studies have estimated potentially significant GWA to be present in $1.5 \%, 1.9 \%$, and $22 \%$ of patients taking proton pump inhibitors undergoing SPECT MPI. ${ }^{1,2,4}$ Also, on review of the nuclear database from St. Luke's Mid America Heart Institute, only 3\% (41/1369) of the patients taking PPIs undergoing SPECT MPI during the past year had an equivocal MPI result, virtually the exact same percentage (117/3952) (3\%) as those patients undergoing SPECT MPI who were not taking a proton pump inhibitor.

This gastric wall radiotracer activity problem is less common with exercise testing than with pharmacologic stress. ${ }^{3}$ Also, the activity is best recognized on review of the rotating planar images, highlighting the importance of meticulous review technique. ${ }^{1}$ Experienced readers can often read around the abnormality. When increased activity does occur in the gastric wall, some of the usual maneuvers to mitigate activity in the biliary tract and stomach content are not helpful. For example, image acquisition time delays can allow biliary and gastric content activity to move through the intestinal tract and 
delayed imaging usually resolves such image interference. Gastric wall activity does not decay faster than cardiac activity, however, as shown in the study by Rose $e t a l^{7}$ Likewise, drinking milk or water is not likely to mitigate the issue either. ${ }^{5}$ The use of attenuation correction is likely to Intensify the problem as removing the attenuating effects of the abdominal wall can make the activity appear brighter. ${ }^{8}$ Adding a non-gated prone image (typically adding an extra 5-6 minutes of scan time) to the patient's supine gated images can often normalize an inferior abnormality seen in the supine view and is worth attempting in patients with interfering gastric wall activity. ${ }^{9}$ The ingestion of carbonated beverages can expand the stomach with carbon dioxide and displace it away from the heart (a maneuver more often used in Japan than in the United States) and likewise seems logical to try. ${ }^{10}$

In extreme cases, repeat stress imaging might be required, and exercise or combined exercise and pharmacologic testing should be considered. Exercise protocols have a lower prevalence of clinically significant stomach uptake compared to stress imaging using vasodilating agents such as Adenosine or Regadenosin. ${ }^{9}$ Therefore, pharmacological stress testing with a modified exercise protocol, if possible, may result in less isotope uptake in the stomach wall. Based on the results of the study by Rose et al, administration of aspirin prior to testing might be helpful, although this has not yet been confirmed. ${ }^{7}$ Based a previous study by Singh et al, cessation of the proton pump inhibitor for three days, while perhaps substituting an $\mathrm{H} 2$ blocker, should decrease the gastric wall activity. ${ }^{3}$ Also, myocardial perfusion PET, which has higher spatial resolution, will likely give better results when GWA is significant on SPECT.

Rose and colleagues are to be congratulated on their insightful paper using a simple observational study design which helps elucidate the mechanism of proton pump inhibition and gastric wall radiotracer activity. Further study is needed to determine whether holding proton pump inhibitors prior to SPECT MPI or administering aspirin to these patients would be necessary or helpful.

\section{Disclosure}

Stephen A. Bloom and Randall C. Thompson declare that they have no financial conflict of interest.

\section{References}

1. Côté C, Dumont M. The clinical meaning of gastric-wall hyperactivity observed on sestamibi cardiac single-photon emission computed tomography. Can Assoc Radiol J 2004;55:178-83.

2. Gholamrezanezhad A, Moinian D, Eftekhari M, Mirpour S, Hajimohammadi $\mathrm{H}$. The prevalence and significance of increased gastric wall radiotracer uptake in sestamibi myocardial perfusion SPECT. Int J Cardiovasc Imaging 2006;22:435-41.

3. Singh H, Mittal BR, Sood A, Bollampally N, Gorla AKR, Dasagrandhi V, Parmar M. Association of use of proton pump inhibitors and $\mathrm{H}_{2}$ antagonists with stomach wall uptake in ${ }^{99 \mathrm{~m}} \mathrm{Tc}-$ methoxy-isobutyl-isonitrile (MIBI) myocardial perfusion imaging. J Nucl Cardiol 2019. https://doi.org/10.1007/s12350-019-01733-9.

4. Mouden M, Rijkee KS, Schreuder N, Timmer JR, Jager PL. Influence of proton-pump inhibitors on stomach wall uptake of 99mTc-tetrofosmin in cadmium-zinc-telluride SPECT myocardial perfusion imaging. Nucl Med Commun 2015;36:143-7. https://doi. org/10.1097/MNM.0000000000000222.

5. Goel S, Bommireddipalli S, DePuey EG. Effect of proton pump inhibitors and $\mathrm{H} 2$ antagonists on the stomach wall in $99 \mathrm{mTc}-$ sestamibi cardiac imaging. J Nucl Med Technol 2009;37:240-3. h ttps://doi.org/10.2967/jnmt.109.065847.

6. Javadi Hamid, Jallalat Sara, Semnani Shahriar, Mogharrabi Mehdi, Nabipour Iraj, Tabib Seyed Masoud, Abbaszadeh Moloud, Assadi Majid. The association of increased stomach wall radiotracer uptake with prolonged use of Omeprazole capsules on myocardial perfusion imaging (MPI) using $99 \mathrm{mTc}$-sestamibi SPECT. Nuclear Med Rev 2013;16:91-4.

7. Rose D, Robinson B, Shanthi K, Lee J. Interaction between the effects of proton pump inhibitors and aspirin on gastric wall sestamibi uptake on myocardial perfusion imaging. J Nucl Cardiol 2020

8. Thompson RC. The problem of radiotracer abdominal activity in myocardial perfusion imaging studies. J Nucl Cardiol 2008;15:159-61.

9. Bloom SA, Meyers K. Reducing radiation to patients and improving image quality in a real-world nuclear cardiology laboratory. J Nucl Cardiol 2017;24:1871-7.

10. Hara M, Monzen H, Futai R, Inagaki K, Shimoyama H, Morikawa $\mathrm{M}$, et al. Reduction of infracardiac intestinal activity by a small amount of soda water in Tc-99m tetrofosmin myocardial perfusion scintigraphy with adenosine stress. J Nucl Cardiol 2008;15:241-5.

Publisher's Note Springer Nature remains neutral with regard to jurisdictional claims in published maps and institutional affiliations. 